\title{
A technique for Cleft Palate Repair
}

\section{Azimov Muhamadjon Ismoilovich, Shomurodov Kahramon Erkinovich}

Tashkent State Dental Institute, Tashkent, Uzbekistan.

\section{Email address:}

azimov@mail.ru (Azimov Muhamadjon Ismoilovich)

\section{To cite this article:}

Azimov Muhamadjon Ismoilovich, Shomurodov Kahramon Erkinovich. A technique for Cleft Palate Repair. Journal of research in health science. Vol. 1, No. 2, 2018, pp. 56-59. DOI 10.26739/2523-1243

\section{doi hittp://dx.doi.org/10.26739/2523-1243/-2018-1-2-9}

\begin{abstract}
: the author has developed a technique of palate repair with carried out by the transection of the soft palate with its elongation and longitudinal suturing of wound. Since 2008 during the treatment of children with congenital cleft palate there have being carried out palatoplasty operations using the method developed by us in the Department of maxillofacial surgery of the clinic of the Tashkent State Dental. This method for ease and technical characteristics justifies itself at the restoration of anatomical and functional qualities of the palate in children with this pathology.
\end{abstract}

Keywords: cleft palate, palatoplasty.

Currently, there are many techniques of surgical treatment of children with congenital cleft palate. But despite this, children's maxillofacial surgeons and patients themselves are not always satisfied with the treatment results because the speech of number of children remains indistinct, with an expressed rhinolalia [1,2,5].

In the surgical treatment of congenital cleft palate (CCP), surgeons should not only close the defect throughout of it, but it is necessary to elongate the palate so that it provided a complete separation of the oropharynx from the nasopharynx and ensured the purity of speech [3].
V. Veau made an attempt to lengthen the soft palate by separating the muscles of the soft palate from a pathological attachment to the hind part of the horizontal palatal plate of the hard. Wardill and Kilner in 1937 developed the socalled "Oxford method" of one-step palatoplasty, which represents the displacement of the palatal mucoperiosteal flaps (V-Y) that give a significant increase in the length of the palate [9].

L. T. Furlow (1976) proposed a method of lengthening of the soft palate with cut and mutually moveable triangular flaps on two levels in opposite directions. The 
medial side of each flap is the edge of nonunion, the end of the lateral cut is the point on the region of the hamulus of the pterygoid process. The flaps on the front foot contain only the mucous membrane, the flaps on the back foot the mucous membrane and muscles of the soft palate. During the mutual displacement muscle stratums are duplicated and contribute to recovery of function of the vellum palatinum. But the orientation of muscle fibers does not conform to normal, and when wide clefts there may be tension in the operative wound on the soft palate and shortening of the palate along (V.S. Dmitrieva and R.L. Lando, 1968; L.V. Kharkov, 1982; A.G. Gulyuk, 2002) [4, 9].

K. Kobus (1984) made an elongation of the palate by V-Y plasty on the border of the hard palate, and the plasty of the soft palate - in 3 months after the first stage [9].

According to many works it is clear that during palatoplastys the authors pay serious attention to the lengthening of palate. The presence of a large number of researches on this issue, confirms its relevance and the need in the further search for solutions to this issue $[2,3,5,6,7]$.

The purpose of the work: to present our method of palatoplasty carried out by the transection of the soft palate with its elongation and longitudinal suturing of wound.

Materials and methods. Since 2008 during the treatment of children with CCP there have being carried out uranoplasty operations using the method developed by us in the Department of maxillofacial surgery of the clinic of the Tashkent State Dental. This method for ease and technical characteristics justifies itself at the restoration of anatomical and functional qualities of the palate in children with this pathology. (Registration number of the patent application on the method of surgical treatment of congenital cleft palate №IAP 20170221).

During last 5 years the surgeons of the Institute have been using actively the proposed method for the treatment of congenital pathology of the palate. So, for the period 2012-2016 177 children with CCP were operated, including 92 (51.9\%) were girls and $85(48 \%)$ boys aged from 9 months to 14 years. In the postoperative period we used antibacterial and restorative therapy. In 2-3 weeks after surgery and then for 3 months there was recommended a complex of physiotherapeutic treatment with a massage of the soft palate.

\section{Below we present the method of surgery} with elongation of the soft palate:

1. Under endotracheal anesthesia we set a dental gag. The baby's head is thrown back maximally.

2. After careful antiseptic treatment nasal-oral region we draw the cutting line with medical marker and infiltrate soft tissues of the palate with anesthetics.

3. Taking with surgical forceps the mucous membrane of the edge of the defect at the level of the connection of the muscles with aponeurosis we dissect the soft palate transversely on both sides for its entire thickness up to 8-12 mm (not always the fragments of the cleft are symmetric, so the length of the incision may vary depending on the individuality of the cleft). The defect takes the form of a rhombus.

4. Cut a strip of mucous membrane along the edges of the cleft of the soft palate from the lower edge of the crosssection to the end of the uvula on both sides. 
Azimov Muhamadjon Ismoilovich, Shomurodov Kahramon Erkinovich. A technique for Cleft Palate Repair

5. Flake off the mucoperiosteal flap (MPF), pull and dissect the neurovascular bundles (NVB) on both sides so that MPF could move freely on a plane to the middle and posterior. Separate nasal mucosa from the hard palate.

6 . We stitch mucose of the nose from the top to the end of uvula with absorbable fiber using inverted suture, on the muscles of the uvula impose nodal suture.
While suturing it happens a displacement of muscles m.veli palatini and m.palatopharyngeus, which are located along the edge of the cleft. These muscles are turn 90 degrees position, then we stitch them "end to end". The defect formed after transection of the soft palate takes a longitudinal view, because of this the palate is lengthened. Complete the operation by suturing the oral mucosa.

\section{Clinical example presented in figure.}

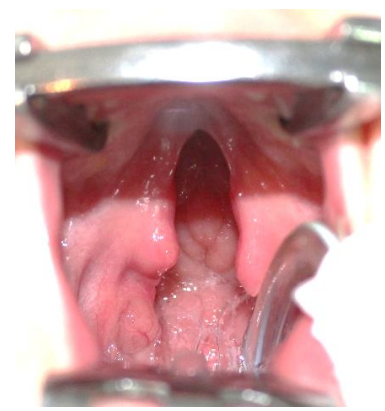

$\mathbf{a}$

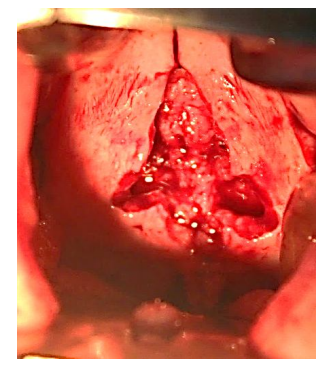

e

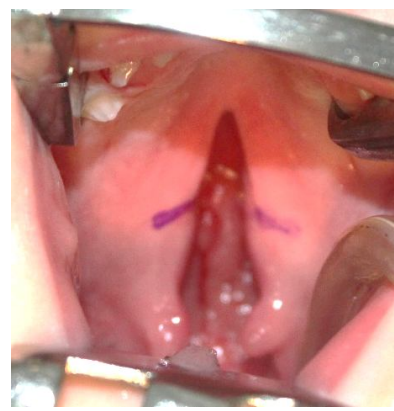

b

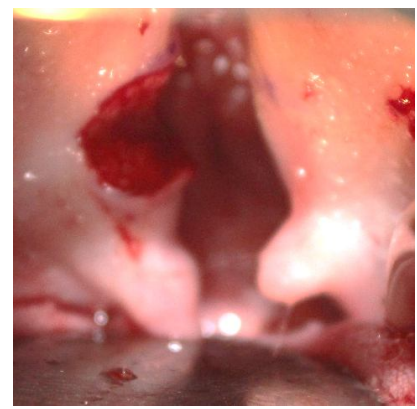

c

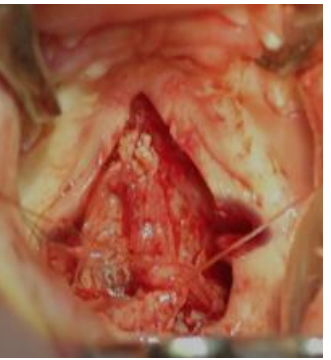

f

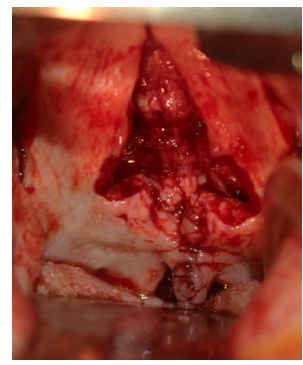

g

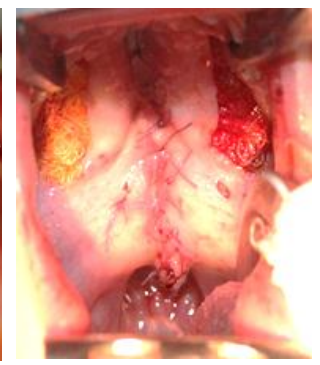

h

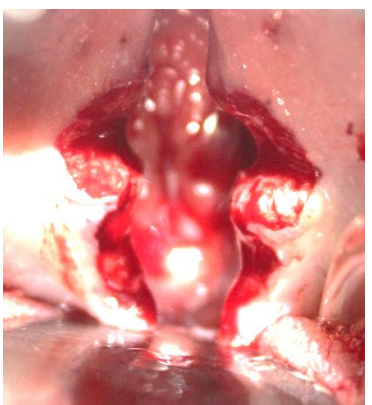

d

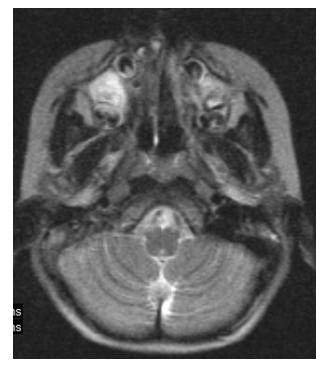

Figure. Clinical example: a - view before surgery: cleft palate of 3a degree; $b$ - the cutting line drawn with medical marker; c - transverse dissections on the soft palate $d$ - transverse dissections of both sides and excision of the mucosal edge of the soft palate and uvula; e - stitching the mucous membrane of the nose from the top to the end of the uvula with inverted suture; $f$ - displacement of muscles m.veli palatinae and m.palatopharyngeus, located along the edge of the cleft and suturing them "end to end"; $g$ - forming the uvula of the soft palate; $h$ - suturing the oral mucous membrane; i - uniform MRI visualization of the palato-pharyngeal ring on 14th day after the surgery.

Thus, our experience of surgical treatment of children with congenital cleft palate with further observation of 4-8year-old children after the surgery, should be estimated as positive and perspective. The influence of the developed method of uranoplasty on the development of the maxilla and the speech quality will be presented in the next publications. 
Journal of research in health science. 2018; 1 (2): 56-59.

\section{References}

1.Bessonov S.N.Surgical treatment of congenital and secondary facial deformations in the cleft lip and palate: dis. ... doctor of medicine.- Smolensk, 2007. - 270 p.

2. Davydov B.N. Surgical treatment of congenital malformations of the face. - Tver: RIO TSMA, 2000. - Pp.160-167.

3. Mamedov Ad.A.Clinical-anatomical classification of congenital cleft lip and palate // Congenital and hereditary pathology of the head, face and neck in children: current issues of comprehensive treatment. - M., 2002. - Pp.155 - 157.

4. Mkrtumyan E.S., Shakhmatova E.V., Lopatin A.V., Yasonov S.A. Palatopharyngeal insufficiency and improving methods for its elimination // Congenital and hereditary pathology of the head, face and neck in children: current issues of comprehensive treatment.- Moscow, 2002.- Pp. 162-163.

5. Khodjamuradov G.M., Shaymonov A.Kh., Tukhtaev F.M. The restoration of the normal anatomy of the muscles of the soft palate in its congenital cleft // Bulletin Of Avicenna. - 2015. - №3 (64). Pp.28-31.

6. Chuikin S.V., Persin L.S., Davletshin N.A.Congenital cleft lip and palate.- Moscow: Medical information Agency, 2008. - 368 p.

7.Le T.M.Aesthetic rehabilitation involving acleft lip and palate // Dent.Today.- 2008.- Vol.27, N 10. - Pp. 124,126, 128.

8. Sommerlad B.C. A technique for Cleft Palate Repair // Plast Reconstr Sur, 2003. - Vol. 112(6).- Pp. $1542-1548$.

9. Sommerlad BC, Mehendale FV. A modified gag for cleft palate repair. Br J Plast Surg. 2000;53:63-4 10.Wyszynski D.F. Cleft lip and palate - from origin to treatment.- Oxford university press, 2002.- Pp. 326-331.

\section{Summary}

Today, there are many methods of surgical treatment of children with congenital cleft palate. But despite this, children's oral surgeons are not always satisfied with the treatment results.

In the surgical treatment of congenital cleft palate surgeons should not only close the defect throughout of it, but it is necessary to elongate the palate so that it provided a complete separation of the oropharynx from the nasopharynx and ensured the purity of speech. The method of uranoplasty developed by us which is carried out by the transection of the soft palate with its elongation and longitudinal suturing of wound should be estimated as a perspective. 